\title{
Organic Chemistry in Meteorites, Comets, and the Interstellar Medium
}

\author{
Oliver Botta $\nmid$ \\ International Space Science Institute, 3012 Bern, Switzerland \\ email: obotta@pop600.gsfc.nasa.gov
}

\begin{abstract}
With the notable exception of those originating on the Moon and Mars, all known meteorites are pieces of objects in the asteroid belt. As such, they have recorded a succession of chemical processes, starting from reactions in the interstellar medium (ISM), followed by reactions that accompanied the formation and evolution of the early solar system, and culminated with reactions during aqueous alteration in the meteorite parent bodies. One of the challenges in meteorite research is to decipher this record and to learn about interstellar formation processes as well as to conditions in the early solar system. The rare class of carbonaceous chondrites contains up to $5 \%$ by weight of organic carbon, most of which is locked in an insoluble macromolecular material and only about $20 \%$ of it is in the form of distinct organic compound classes. The molecular and isotopic data of these organic compounds clearly show an interstellar heritage, but a fraction of these precursors were later modified. For example, the amino acids were probably formed inside the meteorite parent body during the aqueous alteration period from simple molecules such as $\mathrm{HCN}, \mathrm{NH}_{3}$ and carbonyl compounds. However, the CI type carbonaceous chondrites contain a significantly distinct amino acid composition, indicating that there may be other synthetic processes involved. Polycyclic aromatic hydrocarbons (PAHs) are probably the most abundant form of organic carbon in the gas phase in the ISM. PAHs are among the most abundant organic compounds in carbonaceous meteorites, and they have been shown to have a presolar origin. A fraction of these PAHs are present in an extractable form, while the rest is part of the insoluble macromolecular matter. Progress is being made in the understanding of the evolution of this material in relation to aqueous alteration and oxidation. Although the potential of cometary meteorites cannot be ruled out, no such macro-meteorite has been recognized in the meteorite collections. Therefore, the organic composition of comets has been inferred mostly from astronomical observations. Future in-situ investigation of comets with spacecraft such as Rosetta will deliver new data on their organic composition, in particular the non-volatile fraction. However, in order to understand the contributions of different formation processes in primitive solar system objects, the analysis of the organic composition of meteorites remains essential.
\end{abstract}

Keywords. astrobiology - astrochemistry - molecular data - molecular processes - methods: laboratory — comets: general — minor planets — asteroids — solar system: formation stars: AGB and post-AGB

\section{Introduction}

Life on Earth is a consequence of increasing complexity of chemical pathways, which have lead to the formation of stable carbon compounds, themselves being the building blocks of biopolymers and cell components. This evolution started early on in the history of the Universe with the synthesis of the elements heavier than helium in the first generation of stars. During the course of several cycles of star formation and death, these

$\dagger$ Current address: NASA Goddard Space Flight Center, Code 699, Greenbelt, MD 20771, USA 
heavy elements - including carbon, oxygen, nitrogen, sulfur and phosphorous - were redistributed into the interstellar medium (ISM) and provided a basis for the synthesis of complex organic molecules. Even heavier elements such as iron are necessary to form solid planets around stars, so the first few generations of stars are not expected to harbor any type of (terrestrial) planetary system.

\section{Formation of Organic Compounds in Space}

Organic molecules can be formed in different environments in space. In diffuse interstellar clouds, ions are produced via photoionization of small molecules by external UV radiation. Since the UV flux in these regions is rather high, photodissociation occurs in parallel to ionization, preventing the formation of larger molecules. Consequently, only small molecules such as $\mathrm{HCN}, \mathrm{C}_{3} \mathrm{H}_{2}, \mathrm{H}_{2} \mathrm{CO}$ or $\mathrm{CS}$ are present in abundances that are above the detection limit of the current infrared and mm-wave telescopes.

In cold molecular clouds, at temperatures of 10 to $40 \mathrm{~K}$, most of the atoms and molecules are frozen out on grain surfaces. Since $99.9 \%$ of the atoms are hydrogen, the formation of molecular hydrogen on these grain surfaces is the most common process, the details of which are not yet completely understood. However, it has been shown that only surface chemistry can produce molecules such as methane and ammonia, and even methanol, by hydrogen addition reactions in timescales short enough to lead to abundances that are in accordance with the observations. The dust grains act as chemical catalysts for these reactions. On the other hand, more complex molecules form through a chemistry network dominated by gas-phase ion-molecule and neutral-neutral reactions (Herbst 2001), which is driven by galactic cosmic rays that can penetrate throughout the dark cloud. This chemistry results in a distinctive suite of many unsaturated carbon chain molecules, including cyanoplyynes, various cumulene carbenes and chain radicals (Ehrenfreund \& Charnley 2000).

AGB and post-AGB stars are copious producers of carbonaceous dust particles. Interstellar PAHs, and those found in meteorites, probably originated in the cool circumstellar envelopes (CSEs) of carbon stars (Messanger et al. 1998), where their photostability allows them to survive passage out of the CSE into the ISM, ultimately to become incorporated into more complex presolar organic material (Peeters et al. 2003).

Finally, organic molecules could also be produced in the accretionary disk environment around a high- or low-mass star or inside the planetesimals that are being formed in a later stage of planetary system formation. The chemistry in some parts of the disk may be very similar to that in molecular hot cores, where the ices on grains evaporate and more complex molecules are formed in the gas phase driven by thermal heating and the UV radiation coming from the star. In addition, in the accretionary disk, these processes could be repeated several times as the molecules settle back down into the midplane and freeze out again. Aqueous alteration, driven by the radioactive decay of short-lived isotopes inside the meteorite parent bodies, has been proposed to be an early solar system phenomenon which transforms anhydrous minerals into hydrated ones, for example phyllosilicates. In this environment, organic compounds could be formed in aqueous solution from precursor molecules inherited from the interstellar medium.

\section{Investigation Techniques}

\subsection{Astronomical Searches for Molecules in the Interstellar Medium}

Searches for organic molecules in space rely on the cooperation of theoretical modeling, laboratory investigations (for example to obtain the exact infrared or radio frequencies of 
a certain organic molecule in the gas phase or in interstellar ice analogs), and obviously the astronomical observations. Deep searches have been carried out using single-dish telescopes such as the NRAO $12 \mathrm{~m}$ telescope at Kitt Peak, JCMT, CSO and the Green Bank Telescope (GBT). Recently, molecules such as propanal (Hollis et al. 2004), glycolaldehyde (Hollis et al. 2000) and ethyleneglycol (Hollis et al. 2002) have been detected in the interstellar medium. The detection of the simplest amino acid glycine, obtained with the NRAO 12 m telescope, was reported in 2003 (Kuan et al. 2003).

\subsection{Searches for Molecules in Comets}

Our knowledge of the composition of comets is predominantly based on the evaporation of volatile species and thermal emission from dust that occur when bright comets pass through their perihelion. Although no direct observations of molecules on the cometary surface can be obtained with telescopes, the source of the molecules observed in the coma can be inferred from their outgassing properties. It should be noted that although the existence of cometary meteorites cannot be ruled out, no such object has been recognized in the meteorite collections (Campins \& Swindle 1998). Most of the species observed at optical and UV wavelengths are radicals, atoms, and ions that are not directly subliming from the nucleus, but are instead produced in the coma, usually by photolysis of parent molecules, but also by chemical reactions (Bockelée-Morvan et al. 2004). Spectral observations in the mid-IR, in particular by the Infrared Space Observatory (ISO) of comets Hale-Bopp and 103P/Hartley 2, as well as the radio ranges, with SWAS and Odin, have produced a rich database of cometary parent molecules. The first in situ investigation of a cometary nucleus with spacecraft was the pioneering encounter of several interplanetary probes with the active comet Halley in 1986. Only limited information on carbonaceous matter was obtained through mass-spectrometric measurements of Halley's coma. Almost 20 years later, the Stardust spacecraft, while collecting cometary dust particles, encountered comet Wild 2 on January 2, 2004, providing spectacular imagery and mass-spectrometric data Brownlee et al. (2004). Although the particles that will return with Stardust are only of very small mass (1000 particles of $>15 \mu \mathrm{m}$ in size), it may be possible to investigate their organic composition in the laboratory, providing a first direct insight into the refractory organic component of a cometary nucleus. More recently, to investigate the subsurface composition of a comet, the penetrator component of the Deep Impact spacecraft impacted on comet Tempel 1, while the fly-by component recorded the event. Observations of organic and carbonaceous components were carried out during the event in a space- and ground-based campaign. In order to obtain direct information on the refractory organic composition of cometary nuclei, either in-situ analysis of comet nucleus or a sample return mission are necessary. The European Rosetta mission, carrying a small lander that is equipped to carry out a pyrolysis/gas chromatography/mass spectrometry (py-GCMS) analysis of surface material, was launched in 2004 for an encounter with comet 67P/Churyumov-Gerasimenko in 2014.

\subsection{Investigations of Carbonaceous Chondrites}

Meteorites are pieces of asteroids, which are solar system objects most of which failed to grow large enough to ever experience 'planetary' processes like core formation, volcanism or plate tectonics. The chondrite meteorites have near-solar volatile composition and radiometric ages of 4.566 billion years, reflecting their formation during, or shortly after, the birth of the solar system. Within the chondrites, there exists a carbonaceous subclass that contains significant amounts of organic carbon, a characteristic that contributes to a generally dark appearance. The chemistry and mineralogy of carbonaceous chondrites suggests that any organic matter they contain should predate the formation 
of the Earth, therefore reflecting the product of prebiotic chemical evolution in our solar system and the molecular cloud from which it originated. Hence these objects, being the only "macroscopic" samples from beyond the Earth-Moon system, represent a window back in time providing valuable information about the physical and chemical conditions in the early solar system.

\subsection{The Insoluble Component}

The total carbon content of carbonaceous chondrites can be partitioned into soluble and insoluble components. The soluble fraction can be obtained by treating a crushed meteorite sample with a series of solvents of different polarity, which leads to complex mixtures of compounds in the individual extracts. This total soluble fraction of $\mathrm{C} 1$ and C2 chondrites was estimated to contain $30-40 \%$ of the total carbon, but this number is probably an upper limit due to the additional dissolution of inorganic salts in the polar solvents (Cronin93). The insoluble fraction of carbonaceous chondrites is composed of macromolecular matter that is commonly referred to as 'kerogen-like' material. (Kerogen is insoluble macromolecular organic matter, operationally defined as the organic residue left after acid demineralization of a rock.) A protocol using supercritical fluid extraction (SFE), which provides simplified compound mixtures, of meteoritic hydrous pyrolysates in combination with isotope ratio monitoring-GCMS in order to identify and measure the $\delta^{13} \mathrm{C}$ values of individual fragments of macromolecular materials in several carbonaceous has been developed and applied to the Orgueil, Cold Bokkeveld and Murchison meteorites (Sephton et al. 1998; Sephton et al. 2000). The results indicate that the macromolecular materials in these meteorites are qualitatively similar, but also reveal significant quantitative differences that correlate with the aqueous alteration stage of the meteorite.

\subsection{The Soluble Fraction}

The techniques that are used to investigate the soluble organic fraction of carbonaceous chondrites were originally developed for the (unsuccessful) search for organic molecules in returned Apollo lunar samples. However, the fall of the Murchison meteorite in 1969 provided the opportunity to search for amino acids in a fresh fall, which led to the detection of the first extraterrestrial organic compound (Kvenvolden et al. 1970). In the meantime, more than 80 amino acids were identified in Murchison, which is a representative of the CM2 class. Similar amino acid compositions were also found in other CM2 carbonaceous chondrites such as Murray or LEW90500 (see Figure 1; Botta \& Bada 2002). However, investigations of the amino acid composition of the CI carbonaceous chondrites Orgueil and Ivuna revealed a distinct pattern, indicating that the CIs originated from a parent body, or a family of parent bodies, that probably formed from different precursor material than did the CM2 parent bodies (Ehrenfreund et al. 2001).

Although the soluble component of carbonaceous chondrites is only a minor fraction, it contains a suite of organic compounds that has a strong structural link to the origin of life on Earth. Apart from the amino acids already mentioned, a wide variety of Nheterocyclic compounds were also found in meteorites, among them four purines and one pyrimidine (uracil) (Stoks \& Schwartz 1979; Stoks \& Schwartz 1981; Stoks \& Schwartz 1982), compounds that are used in the replicating systems of terrestrial organisms. A persistent issue with these compounds is the lack of unambiguous evidence that they are indeed indigenous to the meteorites rather than terrestrial contamination. However, their structural diversity implies an abiotic origin and provides some means of distinguishing them from their biological counterparts. More recently, a variety of compounds that are related to sugars (sugar alcohols, sugar acids) and the simplest sugar molecule, dihydroxyacetone, were detected in Murchison (Cooper et al. 2001). For two compounds (glycerol 


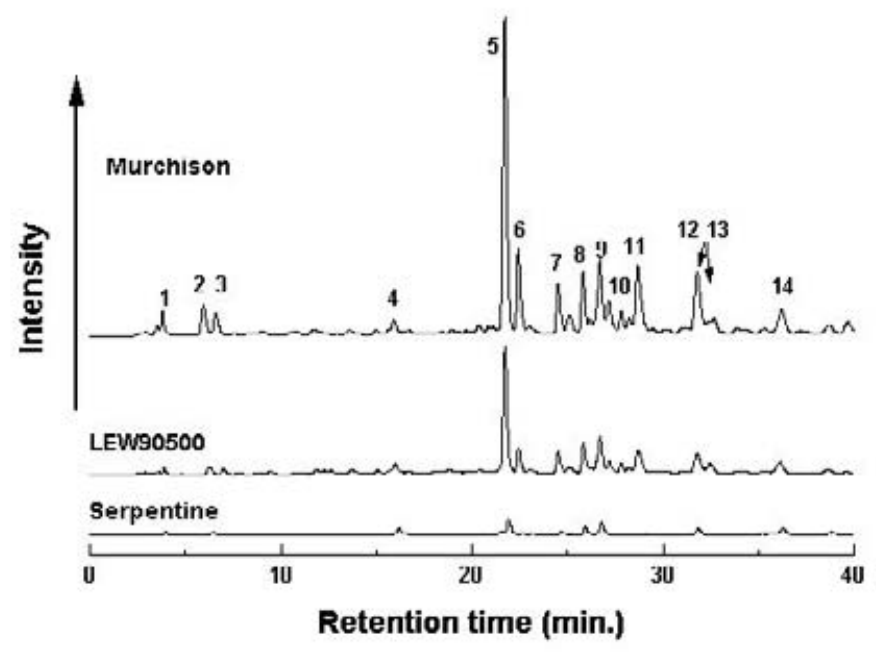

Figure 1. The 0 to $40 \mathrm{~min}$ region (no peaks were found outside this time period) of HPLC traces of $6 \mathrm{M} \mathrm{HCl}$ hydrolyzed hot water extracts of the CM carbonaceous chondrites LEW90500 and Murchison and a serpentine blank after pre-column derivatization with OPA/NAC. Signals: 1 DL-Asp, 2 L-Glu, 3 D-Glu, 4 DL-Ser, 5 Gly, 6 b-Ala, 7 g-ABA, 8 D-Ala, 9 L-Ala, 10 DL-b-ABA, 11 AIB, 12/13 Dl-a-ABA/DL-Isoval, 14 L-Val. Excitiation: 340 nm, Emission: $450 \mathrm{~nm}$.

and glyceric acid), the abundances were determined to be on the same order of magnitude as that for the amino acid glycine. Yet the simplicity and diversity of meteoritic sugar-related compounds contrasts sharply with the high selectivity for sugars found in terrestrial organisms, where deoxyribose and ribose are present in DNA and RNA respectively, glucose is the principle energy source and the highly ordered polysaccharide cellulose is the most abundant organic molecule in the biosphere.

The most abundant class of soluble organic molecules to be found in carbonaceous meteorites are carboxylic acids, at an overall concentration of $\sim 300 \mathrm{ppm}$. Fullerenes were found in the Allende meteorite at a concentration of $>100 \mathrm{ppb}$. Linear amides are the next most abundant class, with concentrations of $>70 \mathrm{ppb}$, followed by amino acids, which are between 15-60 ppb for CM2 and about 5 ppb in CI1 carbonaceous chondrites. For an review on organic compounds found in meteorites see Botta \& Bada (2002).

\section{Formation of Amino Acids and PAHs is Space}

\subsection{Amino Acids in Interstellar Ice Analogs}

Theoretical models on the formation of organic molecules predict the synthesis of compounds such as methanol inside interstellar ices. More complex molecules are formed in gas-phase reactions after these compounds desorb from the refractory core of the grains, either by collisions, by interactions with photons or cosmic rays, or by thermal desorption.

It has been reported that amino acids can form in interstellar ice analogs in the laboratory. Muñoz Caro et al. (2002) showed a chromatogram, obtained from a irradiated ice mixture containing $\mathrm{H}_{2} \mathrm{O} / \mathrm{CH}_{3} \mathrm{OH} / \mathrm{NH}_{3} / \mathrm{CO} / \mathrm{CO}_{2}$, which was warmed up to room temperature after irradiation and the residual hydrolyzed in $6 \mathrm{M} \mathrm{HCl}$, with the identifications 
of a variety of amino acids, including di-amino acids. Although this mixture contained a number of amino acids that are not used in modern biochemistry, and can therefore not be contaminants of any kind, the mixture is dissimilar to amino acid compositions of any carbonaceous chondrite ever analyzed. In particular, some of the most abundant compounds, such $\alpha$-aminoisobutyric acid (AIB) and isovaline, which have been identified in non-acid hydrolyzed meteorite extracts (Botta \& Bada 2002), are not found in the hydrolyzed residue of the interstellar ice analog, indicating that a direct link between the synthetic pathways of these two sources is missing. Since no information about the presence of these amino acids before acid hydrolysis is given in the paper, there is no evidence that the amino acids were actually formed in the ice. However, it is more plausible that the variety of amino acids were formed during acid hydrolysis from precursors that were synthesized in the ice during irradiation. This is in agreement with experiments by Bernstein et al. (2002), who found in their irradiated ice mixture only amino acid precursor before acid hydolysis, which then hydrolyzed to the amino acids glycine, rac-alanine and rac-serine as well as other compounds.

\subsection{Synthesis of Amino Acids in Meteorite Parent Bodies}

The reaction of $\mathrm{HCN}, \mathrm{NH}_{3}$ and carbonyl compounds in the so-called Strecker-cyanohydrin synthesis leads to an equilibrium in aqueous solution between cyanohydrins and aminonitriles. These intermediates then undergo irreversible hydrolysis yielding a mixture of $\alpha$-hydroxy- and $\alpha$-amino acids, respectively. All other conditions being equal, the ratio of these two species in equilibrium depends on the concentration of ammonia in solution. Both $\alpha$-hydroxy and $\alpha$-amino acids are found in the Murchison meteorite, and in some instances, the molar ratios of structurally analogous hydroxy/amino acid pairs are consistent with equilibration of the nitrile precursor at a common ammonia concentration (Peltzer et al. 1984). Further support for the hypothesis that Strecker synthesis is the main pathway for the formation of $\alpha$-amino acids in carbonaceous chondrites is the recent identification of iminodicarboxylicacids in Murchison, which are expected by-products of this reaction (Lerner \& Cooper 2005).

One feature of Strecker synthesis is the fact that the complexity of the side chain of the resulting amino acid is inherited from the the substitution pattern of the carbonyl group. For example, if formaldehyde is the starting product, which only has two hydrogen atoms apart of the carbonyl group, the resulting amino acid glycine has the two hydrogen on the $\alpha$-carbon. Analogously, acetaldehyde will form alanine and acetone will form AIB. Of course, the corresponding $\alpha$-hydroxy acids will also be formed. Hypothetically, one could introduce some of the recently discovered interstellar aldehydes into a Strecker synthesis, since it can be expected that they would react the same way. Propanal, which is a threecarbon aldehyde, will form $\alpha$-amino- $n$-butyric acid, on of the most prominent amino acids in carbonaceous chondrites, and $\alpha$-hydroxy- $n$-butyric acid (see Figure 2). More interestingly, glycolaldehyde, assuming that it is present in the meteorite parent body in the first place, would react to form glyceric acid and serine. While the latter was also found in meteorites (how much of it is indigenous is not clear, however, due to incomplete separation of the enantiomers), the former is one of the sugar-related compounds found recently by Cooper et al. (2001) in Murchison. Therefore, perhaps Strecker synthesis may be a synthetic pathway to form not even amino acids, but also other astrobiologically relevant compounds in the meteorite parent bodies.

It should be noted here that the synthesis of a variety of amino acids such as $\beta$-alanine, $\beta$-amino- $n$-butyric acid and $\gamma$-amino- $n$-butyric acid cannot be explained by Strecker pathway. Alternatively, $\beta$-amino acids could be synthesized through Michael-addition of ammonia to substituted $\alpha, \beta$-unsaturated nitriles followed by hydrolysis, a mechanism 


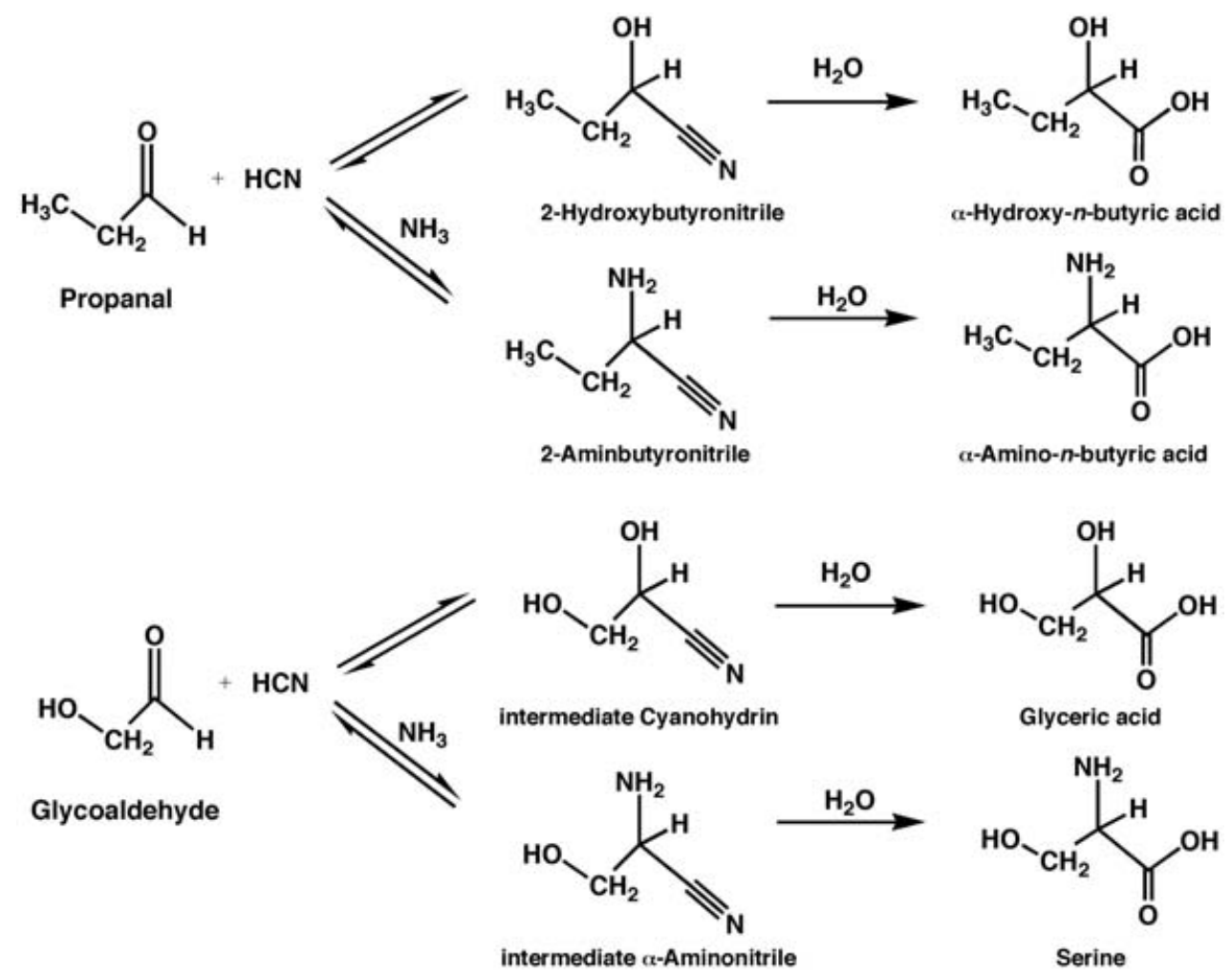

Figure 2. Theoretical synthetic reaction schemes for astrobiologically interesting compounds via Strecker-cyanohydrin pathway from aldehydes detected in the interstellar medium. Top: Formation of $\alpha$-amino- $n$-butyric acid and the corresponding $\alpha$-hydroxy acid from propanal. Bottom: Formation of glyceric acid and $\alpha$-serine from glycolaldehyde

proposed for the synthesis of $\beta$-alanine in spark discharge experiments. The starting material for the synthesis of $\beta$-alanine for this process would be acrylonitrile, a molecule that has been detected in the ISM. Also, the recently detected di-amino acids in Murchison (Meierhenrich et al. 2004) are not simple products of Strecker chemistry, indicating that other synthetic pathways need to be explored to explain the diverse suite of amino acids identified in carbonaceous chondrites.

\subsection{Polycyclic Aromatic Hydrocarbons (PAHs)}

As already mentioned earlier, the major source for PAHs in space are outflows of of carbon-rich AGB, post-AGB and protoplanetary nebulae (PPNe). Due to the chemical and physical conditions in the photospheres and winds of these stars, which resemble those in sooting flames, it is speculated that the PAHs are intermediates towards the production of macromolecular assemblies of carbon and carbon dust particles (e.g., Cherchneff et al. 1992). Recent ISO observations have led to detection of benzene in the PPNe CRL 618 (Cernicharo et al. 2001), the discovery of the first aromatic compound outside the solar system. In carbonaceous chondrites, free PAHs have been investigated using two-step laser mass spectroscopy (Hahn et al. 1988). However, most of the aromatic compounds in meteorites are locked in the macromolecular material, which is difficult to access analytically. There are basically two ways to obtain information about the structure of this material. The first is the use of solid state ${ }^{1} \mathrm{H}$ and ${ }^{13} \mathrm{C}$ Nuclear Magnetic Resonance (NMR) spectroscopy, which allows the determination of functional 
group abundances in the sample. Gardinier et al. (2000) re-examined the Murchison and Orgueil macromolecular materials and determined the amount of aromatic carbon to be between 69 and $78 \%$ in Orgueil and 61 and $67 \%$ in Murchison. Subsequent studies by Cody and coworkers (e.g., Cody \& Alexander 2005) have refined these numbers to be between 61 and $66 \%$ for both CI and CM-type meteorites, 48 to $52 \%$ for CR2, and 79 to $83 \%$ in the unusual Tagish-Lake meteorite. Generally, these results suggest that the primary differences observed in the macromolecular material of different meteorite classes most likely reflect processes that occurred on the meteorite parent body. The second analytical method to investigate the macromolecular material involves hydrous pyrolysis, where the sample remains in in contact with water during pyrolysis, followed by GCMS. Qualitatively, hydrous pyrolysates of different meteorites were found to be very similar, indicating that macromolecular materials of different classes of meteorites are made up of essentially the same units (Sephton et al. 1998). More recently, $\mathrm{H}_{2}$-pyrolysis has been applied to investigate the composition of the meteorite macromolecular material (Sephton et al. 2004), releasing significant amounts of high molecular weight PAHs with varying degrees of alteration. The largest $\mathrm{PAH}$ identified by this method is the seven-ring PAH coronene $\left(\mathrm{C}_{24} \mathrm{H}_{12}\right)$, which is about half the size expected for interstellar PAHs and components of the carbonaceous dust (Pendleton \& Allamandola 2002). This result, and the deduction that even larger entities should be present in the residue, partly reconciles the apparent discrepancy between the meteoritic and interstellar aromatic components of the organic material.

\section{Summary}

A combination of interstellar ice processing at extremely low temperatures, circumstellar formation pathways and gas-phase chemistry results in the distribution of organic molecules observed in the interstellar medium. During formation of the solar system, some interstellar organic compounds were incorporated into growing planetesimals, but most of them were destroyed by shocks, radiative and thermal processes that occurred in the very early phases. Comets, being the most pristine, though not completely unmodified objects in the solar system, are a key to the understanding of the composition of the presolar nebula. However, astronomical observations are limited to organic volatiles. The detection of refractory organic molecules requires space missions that are capable of in situ analysis or sample return. The organic composition of carbonaceous chondrites is a result of a combination of interstellar synthetic processes and secondary processing of these precursors in the meteorite parent body. Therefore, in order to understand the contributions of different formation processes of organic compounds before, during and after the formation of the solar system, the analysis of the organic composition of meteorites, both in the soluble and the macromolecular fractions, remains an essential analytical tool in the future.

\section{References}

Allamandola, L.J., Tielens, A.G.G.M., \& Barker, J.R. 1989, Ap. J. Suppl. 71, 733

Bernstein, M.P., et al. 2002, Nature 416, 401

Bockelée-Morvan D., Crovisier, J., Mumma, M., \& Weaver, H.A. 2004, in Comets II (University of Arizona Press), p. 391

Botta, O. \& Bada, J.L. 2002, Lunar and Planetary Science XXXIII, Abstract \#1391, Lunar and Planetary Institute, Houston (CD-ROM)

Botta, O. \& Bada, J.L. 2002, Surv. Geophys. 23, 411

Brownlee, D., et al. 2004, Science 304, 1764 
Campins, H. \& Swindle, T.D. 1998, Meteorit. Planet. Sci. 33, 1201

Cernicharo, J., et al. 2001, Ap. J. 546, L123

Cherchneff, I., Barker, J.R., \& Tielens, A.G.G.M. 1992, Ap. J. 401, 269

Cody, G.D. \& Alexander, C.M.O'D. 2005, Geochim. Cosmochim. Acta 69, 1085

Cooper, G., et al. 2001, Nature 414, 879

Cronin, J.R. \& Chang, S. 1993, in The Chemistry of Life's Origins (Kluwer, Dordrecht), p. 209

Ehrenfreund, P. \& Charnley, S.B. 2000, ARAA 38, 427

Ehrenfreund, P., et al. 2001, Proc. Natl. Acad. Sci. USA 98, 2138

Gardinier, A., et al. 2000, Earth Planet. Sci. Lett. 184, 9

Hahn, J.H., et al. 1988, Science, 239, 1523

Herbst, E. 2001, Chem. Soc. Rev. 30, 168

Hollis, J.M., et al. 2000, Ap. J. 540, L107

Hollis, J.M., et al. 2002, Ap. J. 571, L59

Hollis, J.M., et al. 2004, Ap. J. 610, L21

Kuan, Y.-J., et al. 2003, Ap. J. 593, 848

Kvenvolden, K., et al. 1970, Nature 228, 923

Lerner, N.R. \& Cooper, G.W. 2005 Geochim. Cosmochim. Acta 69, 2901

Meierhenrich, U.J., et al. 2004 Proc. Natl. Acad. Sci. USA 101, 9182

Messenger, S., et al. 1998, Ap. J. 502, 284

Mumma, M.J., et al. 2003, Adv. Space Res. 31, 2563

Muñoz Caro, G.M., et al. 2002, Nature 416, 403

Peeters, Z., et al. 2003, Ap. J. 593, 129

Peltzer, E.T., et al. 1984, Adv. Space Res. 4, 69

Pendleton, Y.J. \& Allamandola, L.J. 2002, Ap. J. Suppl. 138, 75

Sephton, M.A., Pillinger, C.T., \& Gilmour I. 1998, Geochim. Cosmochim. Acta 62, 1821

Sephton, M.A., Pillinger, C.T., \& Gilmour I. 2000, Geochim. Cosmochim. Acta 64, 321

Sephton, M.A., et al. 2004, Geochim. Cosmochim. Acta 68, 1385

Stoks, P.G. \& Schwartz, A.W. 1979, Nature, 282, 709

Stoks, P.G. \& Schwartz, A.W. 1981, Geochim. Cosmochim. Acta, 45, 563

Stoks, P.G. \& Schwartz, A.W. 1982, Geochim. Cosmochim. Acta, 46, 309

\section{Discussion}

IRVINE: Can we constrain the length of the acqueous phase in carbonaceous chondrites?

BoтtA: Strontium and Manganese/Chromium isotopic dating established aqueous alteration as a very early process on the meteorite parent body, occurring only 10 to $20 \mathrm{Myr}$ after the time of formation of the oldest known solar nebula condensates (Endress et al. 1996; Nature 379, 701).

ELLINGER: How can we be sure of the $\mathrm{pH}$ inside meteorites?

BotтA: The $\mathrm{pH}$ in meteorite parent bodies has been estimated from the composition of the fluid (DuFresne \& Anders 1962; Geochim. Cosmochim. Acta 26, 1085). The value is estimated to be in the range $6<\mathrm{pH}<8$. 


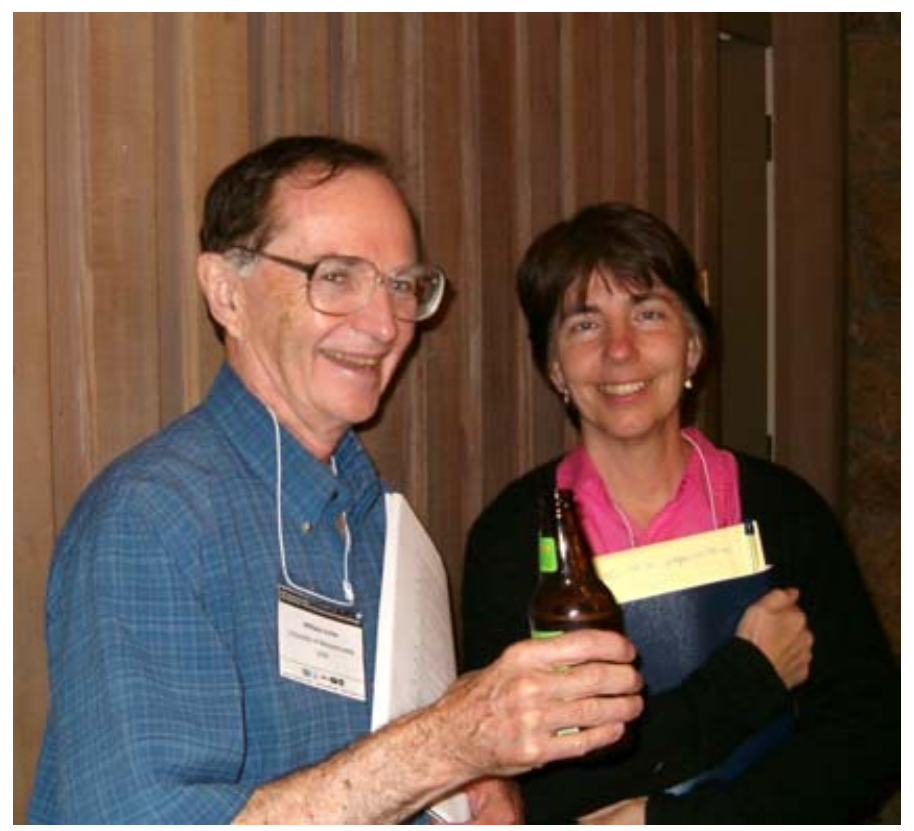

Photo: E. Herbst 